Article

\title{
Robust PD-Type Iterative Learning Control of Discrete Linear Repetitive Processes in the Finite Frequency Domain
}

\author{
Lei Wang ${ }^{1, *\left(\mathbb{B}, \mathrm{Mu} \mathrm{Li}^{2,(1)} \text { and Huizhong Yang }\right.}{ }^{1, * \mathbb{D}}$ \\ 1 Key Laboratory of Advanced Process Control for Light Industry of Ministry of Education, \\ Jiangnan University, Wuxi 214122, China \\ 2 Mechatronics Engineering Group, University of Southampton, Southampton SO17 1BJ, UK; m.li@soton.ac.uk \\ * Correspondence: 7151905015@vip.jiangnan.edu.cn (W.L.); yhz@jiangnan.edu.cn (H.Y.)
}

Received: 13 May 2020; Accepted: 15 June 2020; Published: 18 June 2020

check for updates

\begin{abstract}
This paper studies a robust iterative learning control design for discrete linear repetitive processes in the finite frequency domain. Firstly, the state-space model of the iterative learning process is deduced. Then the dynamic performance condition of the control system in the finite frequency domain is derived by combining it with the stability theory of discrete linear repetitive processes. The system performances in the finite frequency domain are then transformed into the corresponding solutions of the linear matrix inequality by using the generalised KYP lemma. Finally, an integrated state feedback PD-type iterative learning control strategy is proposed. The robust control problem with norm-bounded uncertainty and convex polyhedral uncertainty are also considered in this paper. The simulation of the injection velocity in injection molding verified that the proposed methods in this paper are more effective than the P-type state feedback iterative learning control algorithm.
\end{abstract}

Keywords: finite frequency domain; discrete linear repetitive processes; PD-type iterative learning control; generalized KYP lemma; linear matrix inequality; robust control

\section{Introduction}

Iterative learning control is an intelligent control method with learning and memory function, applicable to repeated operation processes. The fundamental theory is to correct the current control signal based on the previous control experience and output error of the system, in order to improve system performance and eventually realise the complete tracking task in a finite time interval $[1,2]$. The control system has a self-learning function and the structure of its controller is relatively simple. It merely requires some knowledge of dynamic characteristics and online calculation. Therefore, this technology has been widely used in the robot, power electronics, and chemical industries [3-6].

A linear repetitive process is a special two-dimensional system. It is unique in that it has a series of repetitive operations, each of which is done in a fixed finite time. The system is reset before and after each operation, so the result of the current output will take effect on the next operation, thus directly affecting the secondary output $[7,8]$. Each operation process is called a channel of linear repeat process, and the fixed finite time is the channel length. The self-learning mechanism in linear repetition of iterative learning control processes and linear repetitive processes are essentially consistent. In its run time, the system information not only propagates along the channel (along the pass), but also between the channels (pass to pass) that is, a two-dimensional system with dynamic behaviour in two independent directions [9]. In the framework of two-dimensional system theory, iterative learning control for linear repetitive processes has also attracted great interest among researchers. In reference [10], a new iterative learning control algorithm is proposed for a class of discrete-time linear 
systems with a uniform rank greater than 1, and sufficient conditions for the closed-loop system to meet the steady-state performance are given in the form of LMI (linear matrix inequality). The simulation and an experiment with a truss robot verified the effectiveness of this method. In order to solve the actuator failure problem of discrete linear repetitive processes with convex polyhedral uncertainties, a robust fault-tolerant iterative learning controller is designed by using the parameter-dependent Lyapunov function in reference [11], which guarantees the fault-tolerant stability of the learning process and widens the uncertainty range. Furthermore, in reference [12], a robust fault-tolerant iterative control strategy is studied based on the output feedback information for the dual-rate sampling linear repetitive process with output delay. The stability conditions of the closed-loop system are derived by using the stability theory of linear repetitive process and LMI tools.

In fact, the above research work is carried out in the time domain, and the frequency domain analysis method is also a basic research method for iterative learning control of the linear repetitive process. The purpose of control system design is to find a suitable iterative learning controller, so that the closed-loop system can meet some performance indicators (stability, robustness, etc.). From the frequency domain point of view, to meet these performance indexes actually requires the whole closed-loop system to have appropriate frequency domain response characteristics (amplitude-frequency characteristics, etc.). As the reference trajectory of the system in the iterative learning control process often belongs to a specific frequency band and some control performance indexes are also frequency-sensitive, consider the characteristics of the controlled system. Many problems in control engineering can be attributed to the analysis and synthesis of the finite frequency performance index. In two-dimensional theory, the generalised KYP lemma provides a new perspective for researchers to explore the analysis and synthesis of the finite frequency domain from the state-space perspective. It successfully establishes the relationship between the frequency-domain inequality based on transfer function and the time-domain condition (linear matrix inequality) equivalence. Therefore, the generalised KYP lemma becomes an effective tool to deal with the iterative learning control problem of linear repetitive processes in the finite frequency domain. For the discrete repetitive process with norm-bounded uncertainty, the iterative learning controller designed in reference [13] can ensure the convergence of the control system in the iterative direction in the limited frequency domain, combined with the structural characteristics of the repetitive process. In reference [14], based on the stability theory of linear repetitive process, an iterative learning control algorithm combining output feedback controller, which is stable in time domain, and a feed-forward controller, which is monotonically convergent along the iterative direction, are designed. In the limited frequency domain, the sufficient conditions for the existence of the controller are analysed by using generalised KYP lemma and LMI technology. The P-type iterative learning control algorithm used in these studies analyses the control performance of linear repetitive processes in a limited frequency domain.

In order to improve the convergence speed of the learning process, this paper studies the robust iterative learning control of discrete linear repetitive processes in the limited frequency domain based on the stability theory of discrete linear repetitive processes. Iterative learning control (ILC) is a new control strategy to improve the instantaneous response and process tracking performance for controlled objects with repetitive motion. ILC has the advantages of simple controller form, small on-line computation, strong adaptability and easy implementation, less knowledge of dynamic characteristics and complete tracking of controlled objects. ILC is of great value in the study of nonlinear, strongly coupled, difficult modelling systems such as robots. The iterative learning controller has a similar structure to the PID instrument. The PID control system considers to adjust the error in the current time axis direction. Although it can track the given trajectory, the system takes a long time to track the expected trajectory. The iterative learning control system is the deviation control system generated between batches. After learning, the system can fully track the expected trajectory.

Through the integrated state feedback PD-type iterative learning control integrated design method, the state-space model of the iterative learning process is derived, and the dynamic performance condition of the control system in the finite frequency domain is obtained. Then, by using the 
generalised KYP lemma, the finite frequency performance of the system is transformed into the corresponding matrix inequality to solve the problem. At the same time, the problem of robust control with norm-bounded uncertainty and convex polyhedral uncertainty in the system model matrix is also studied. Finally, in the simulation of the injection velocity in injection molding, compared with the state feedback P-type iterative learning control algorithm, the effectiveness and superiority of the design method proposed in this paper are verified.

Throughout this paper:

- $\quad$ for matrix $X, X^{T}$, and $X^{\perp}$ are used to represent its transposition and orthogonal complement;

- $\quad X>0$ and $X<0$ are used to represent that matrix $X$ is positive definite and negative definite;

- $\quad I$ and 0 represent unit matrix and zero matrix of appropriate dimension;

- the symbol ${ }^{\text {*’ }}$ denotes block entries in symmetric matrices;

- $\operatorname{sym}(A)=A+A^{T}$ indicates the Hermitian part of the matrices;

- $\quad \operatorname{diag}(\bullet)$ denotes the diagonal matrix;

- $\quad \rho(\bullet)$ and $\bar{\sigma}(\bullet)$ denote the spectral radius and maximum singular value, respectively, of their matrix arguments.

\section{Background and Problem Formulation}

\subsection{Discrete Linear Repetitive Processes}

The state-space model of the standard discrete linear repetitive process [15] has the following form under $0 \leq t \leq \alpha-1, k \geq 0$ :

$$
\left\{\begin{array}{l}
x(t+1, k+1)=A_{d} x(t, k+1)+B_{d} u(t, k+1)+B_{d 0} y(t, k) \\
y(t, k+1)=C_{d} x(t, k+1)+D_{d} u(t, k+1)+D_{d 0} y(t, k)
\end{array}\right.
$$

where:

- $\quad x(t, k) \in R^{n}$ is the state vector;

- $u(t, k) \in R^{m}$ is the control input vector;

- $y(t, k) \in R^{l}$ is the pass profile (output) vector.

To implement this process, set the boundary conditions of the system as follows:

$$
\begin{aligned}
& x(0, k+1)=d_{k+1}, \quad k \geq 0 \\
& y(t, 0)=f(t), \quad 0 \leq t \leq \alpha-1
\end{aligned}
$$

where $d_{k+1}$ and $f(t)$ are vector functions.

The unique control problem for repetitive processes is that the sequence of pass profiles (outputs) generated can contain oscillations that increase in amplitude in the pass-to-pass direction $(k)$. Hence, the stability theory for linear repetitive processes [15] demands that a bounded initial pass profile (outputs) produces a bounded sequence of pass profiles $\{y(k)\}$ and in its strongest form demands this property holds for all possible values of the finite pass length.

Lemma 1 ([16]). Suppose that the pair $\left\{A_{d}, B_{d 0}\right\}$ is controllable and the pair $\left\{C_{d}, D_{d 0}\right\}$ is observable.

The discrete linear repetitive process described by (1) and (2) is stable along the pass if, and only if:

1. $\rho\left(D_{d 0}\right)<1$

2. $\rho\left(A_{d}\right)<1$

3. all eigenvalues of $G_{d}(z)=C_{d}\left(z I-A_{d}\right)^{-1} B_{d 0}+D_{d 0}, \forall|z|=1$ have modulus strictly less than unity.

The standard discrete linear system stability test can be used to verify that each of the conditions in the results are consistent. The first two conditions of Lemma 1 are used to test the stability of standard linear systems. For the third condition, we need to calculate all the points on the unit circle. 
There are computations here, so some problems may occur.Then, in general, this condition requires that when designing the control law of the control system, the frequency gain is required to attenuate over the whole frequency range. However, in practical engineering, in many cases, the system is not required to work in the full frequency domain. Therefore, in some cases, the analysis within a specific frequency range is more in line with the practical requirements than the analysis within the entire frequency range, especially in the iterative learning control area where the reference signal only has the main frequency content within a limited range. In this paper, a new design algorithm is proposed to study the application of frequency attenuation in the finite frequency domain. The next section further considers the robustness of the system in this paper. First, the generalized KYP lemma is proposed.

Lemma 2 ([15]). For a discrete linear time-invariant system with a transfer-function matrix $G_{d}(z)$ and a frequency response matrix $G_{d}\left(e^{j \theta}\right)=C_{d}\left(e^{j \theta} I-A_{d}\right)^{-1} B_{d}+D_{d}$, the following inequalities are equivalent:

1. the frequency domain inequality

$$
\left[\begin{array}{c}
G_{d}\left(e^{j \theta}\right) \\
I
\end{array}\right]^{T} \Pi\left[\begin{array}{c}
G_{d}\left(e^{j \theta}\right) \\
I
\end{array}\right]<0, \forall \theta \in \Theta
$$

where $\Pi$ is a given real symmetric and $\Theta$ denotes the frequency ranges in Table 1:

Table 1. System frequency value range.

\begin{tabular}{cccc}
\hline & Low-Frequency Range & Middle-Frequency Range & High-Frequency Range \\
\hline$\Theta$ & $|\theta|<\theta_{i}$ & $\theta_{1}<\theta<\theta_{2}$ & $|\theta| \geq \theta_{h}$ \\
\hline
\end{tabular}

2. the LMI

$$
\left[\begin{array}{cc}
A_{d} & B_{d} \\
I & 0
\end{array}\right]^{T} \Xi\left[\begin{array}{cc}
A_{d} & B_{d} \\
I & 0
\end{array}\right]+\left[\begin{array}{cc}
C_{d} & D_{d} \\
0 & I
\end{array}\right]^{T} \Pi\left[\begin{array}{cc}
C_{d} & D_{d} \\
0 & I
\end{array}\right]<0
$$

where $Q>0, P=P^{T}$, and the matrix $\Xi$ is partitioned as

$$
\Xi=\left[\begin{array}{l|l}
\Xi_{11} & \Xi_{12} \\
\hline \Xi_{12}^{T} & \Xi_{22}
\end{array}\right]
$$

and specified as follows:

$$
\Xi=\left\{\begin{array}{c|c}
{\left[\begin{array}{c|c}
-P & Q \\
Q & P-2 \cos \left(\theta_{l}\right) Q
\end{array}\right]} & \text { if }|\theta|<\theta_{l} \\
{\left[\begin{array}{c|c}
-P & e^{j\left(\theta_{1}+\theta_{2}\right) / 2} Q \\
\hline e^{-j\left(\theta_{1}+\theta_{2}\right) / 2} Q & P-2 \cos \left(\left(\theta_{2}-\theta_{1}\right) / 2\right) Q
\end{array}\right]} & i f \theta_{1} \leq \theta \leq \theta_{2} \\
{\left[\begin{array}{c|c}
-P & -Q \\
\hline-Q & P+2 \cos \left(\theta_{h}\right) Q
\end{array}\right]} & i f|\theta|>\theta_{h}
\end{array}\right.
$$

Remark 1. As emphasised in [15], the introduction of generalized KYP lemma makes it possible to study iterative learning control in the frequency domain, and a key step in the study of iterative learning control is the design of control laws that allow designers to avoid the use of frequency grids and weighted filters. In practical engineering, because some systems operate within a certain frequency range, the designer can emphasize a particular frequency range. Since it has been pointed out in the references that bandwidth has the greatest influence on the convergence rate of tracking errors, the main focus of our study is the low frequency range. For better performance, consider some bandwidth over frequencies. In the high frequency range far above the reference signal bandwidth, especially far above the reference signal bandwidth, there will often be noise and interference, which cannot be effectively attenuated by ILC law. 
Lemma 3 ([17]). Given a symmetric matrix $\Psi \in R^{p \times p}$ and two matrices $\Lambda, \Sigma$ of column dimension $p$, there exists a matrix $W$ such that the following inequality holds:

$$
\Psi+\operatorname{sym}\left\{\Lambda^{T} W \Sigma\right\}<0
$$

if, and only if, the following two projection inequalities with respect to $W$ are satisfied:

$$
\Lambda^{\perp T} \Psi \Lambda<0, \quad \Sigma^{\perp T} \Psi \Sigma<0
$$

where $\Lambda^{\perp}$ and $\Sigma^{\perp}$ are arbitrary matrices whose columns from a basis of null spaces of $\Lambda$ and $\Sigma$ respectively.

Lemma 4 ([18]). Given matrices $\Gamma=\Gamma^{T}, X, Y, \Delta$ of compatible dimensions, then:

$$
\Gamma+X \Delta Y+Y^{T} \Delta^{T} X^{T}<0
$$

for all $\Delta$ satisfying $\Delta^{T} \Delta \leq I$ if, and only if, there exists a scalar $\varepsilon>0$ such that:

$$
\Gamma+\varepsilon^{2} X X^{T}+\varepsilon^{-2} Y^{T} Y<0
$$

\subsection{System Description}

This paper considers a linear discrete system,setting as:

$$
\left\{\begin{array}{l}
x(t+1, k)=A x(t, k)+B u(t, k) \\
y(t, k)=C x(t, k)
\end{array}\right.
$$

where:

- $\quad$ on trial $k \geq 0$;

- $\quad$ repetition period $0 \leq t \leq T-1$;

- $\quad x(t, k) \in R^{n}$ is the state vector;

- $u(t, k) \in R^{m}$ is the control input vector;

- $y(t, k) \in R^{l}$ is the control input vector.

The boundary conditions $x(0, k)=x_{0}$ represent the initial condition of the system at the $k$ time. $A$, $B$ and $C$ are corresponding system parameter matrices of appropriate dimensions, and $C B \neq 0$. In the time direction, a standard forward operator $z$ is defined [19]:

$$
z x(t, k)=x(t+1, k)
$$

For details of how the z-transform can be applied over the finite pass length without errors arising from the basic definition being over an infinite interval, see reference [19].

Therefore, assuming that the coefficient $\{A, B\}$ of system (11) is controllable and coefficient $\{C, A\}$, and is considerable, it can be represented by the following transfer function matrix:

$$
G(z)=C(z I-A)^{-1} B
$$

In the iterative learning process of the system (11), the expected trajectory is $y_{d}(t)$; meanwhile, the tracking error of the system at $k$ is defined as:

$$
e(t, k)=y_{d}(t)-y(t, k)
$$

In the iterative learning controller design problem of linear repetitive processes, the sum of the previous batch of inputs and the correction term is used to construct the equivalent batch of controller inputs. 
For system (11), consider the following typical iterative learning control law as:

$$
u(t, k+1)=u(t, k)+\Delta u(t, k+1), k \geq 0
$$

where $\Delta u(t, k+1)$ denotes the correction term to be designed.

To facilitate the analysis, intermediate variables are introduced:

$$
\eta(t+1, k+1)=x(t, k+1)-x(t, k)
$$

The new law of PD-type iterative learning control with state feedback is designed as follows:

$$
\Delta u(t, k+1)=K_{1} \eta(t+1, k+1)+K_{2} e(t, k)+K_{3} e(t+1, k)
$$

where $K_{1}, K_{2}$ and $K_{3}$ are matrices to be designed.

The correction term in this ILC design uses the current iteration to generate state feedback control signals and the PD-type learning items generated in the previous iteration. The state feedback control signal is used to improve the transient response in the time direction. The purpose of the PD-type learning term is to improve the error convergence rate of the learning process.

From Equations (11)-(17), we have:

$$
\begin{aligned}
\eta(t+1, k+1) & =A x(t-1, k+1)+B u(t-1, k+1)-A x(t-1, k)+B u(t-1, k+1) \\
& =\left(A+B K_{1}\right) \eta(t, k+1)+B K_{2} e(t-1, k)+B K_{3} e(t, k) \\
e(t, k+1) & =e(t, k)-[y(t, k+1)-y(t, k)]=e(t, k)-C \eta(t+1, k+1) \\
& =-C\left(A+B K_{1}\right) \eta(t, k+1) \\
& =-C\left(A+B K_{1}\right) \eta(t, k+1)-C B K_{2} e(t-1, k)+\left(I-C B K_{3}\right) e(t, k)
\end{aligned}
$$

In Equation (18), the transfer function matrix from $e(t, k)$ to $\eta(t+1, k+1)$ is

$$
F(z)=\left[z I-\left(A+B K_{1}\right)\right]^{-1}\left(B K_{2}+z B K_{3}\right)
$$

Assuming $z I-\left(A+B K_{1}\right)$ is a nonsingular matrix, then:

$$
\left[z I-\left(A+B K_{1}\right)\right]^{-1}\left[z I-\left(A+B K_{1}\right)\right]=I
$$

Further, get:

$$
z\left[z I-\left(A+B K_{1}\right)\right]^{-1}=I+\left[z I-\left(A+B K_{1}\right)\right]^{-1}\left(A+B K_{1}\right)
$$

Hence:

$$
F(z)=\left[z I-\left(A+B K_{1}\right)\right]^{-1} B K_{2}+B K_{3}+\left[z I-\left(A+B K_{1}\right)\right]^{-1}\left(A+B K_{1}\right) B K_{3}
$$

Also, the tracking error on pass $\mathrm{k}$ is given in the $\mathrm{z}$ domain by:

$$
E_{k}(z)=Y_{d}(z)-Y_{k}(z)=Y_{d}(z)-G(z) U_{k}(z)
$$

where $Y_{d}(z)$ is the $z$ transformation of the expected trajectory, so $E_{k+1}(z)$ can be written as:

$$
E_{k+1}(z)=E_{k}(z)-\left[Y_{k+1}(z)-Y_{k}(z)\right]=G_{e}(z) E_{k}(z)
$$

By combining (19) and (20), $G_{e}(z)$ can be written as:

$$
G_{e}(z)=I-C F(z)=-C\left[z I-\left(A+B K_{1}\right)\right]^{-1}\left[B K_{2}+\left(A+B K_{1}\right) B K_{3}\right]+\left(I-C B K_{3}\right)
$$


If, and only if, all the eigenvalues of $G_{e}(z)$ are strictly less than 1 , the following formula is true:

$$
\rho\left(G_{e}\left(e^{j \theta}\right)\right)<1, \forall \theta \in[-\pi, \pi]
$$

So, the tracking error of the system converges at $k \rightarrow \infty$.

Most ILC schemes exhibit poor transients during the convergence process even if Inequation (27) is satisfied. Specifically, the tracking error may grow over the initial pass before converging as $k \rightarrow \infty$. To avoid this problem, a stronger convergence criteria are required and, in particular, monotonic pass-to-pass error convergence holds if, and only if:

$$
\sigma\left(G_{e}\left(e^{j \theta}\right)\right)<1, \forall \theta \in[-\pi, \pi]
$$

In common with much of the ILC literature, Equation (28) is used from this point onwards. Moreover, since

$$
\sigma\left(G_{e}\left(e^{j \theta}\right)\right)<1 \Leftrightarrow\left\|G_{e}\left(e^{j \theta}\right)\right\|_{\infty}<1, \forall \theta \in[-\pi, \pi]
$$

Equation (28) can be obtained:

$$
\|e(t, k+1)\|_{2} \leq\left\|G_{e}\left(e^{j \theta}\right)\right\|_{\infty}\|e(t, k)\|_{2}
$$

where $\|\bullet\|_{2}$ is $\ell_{2}$ norm.

Then we have:

$$
\|e(t, k)\|_{2} \leq\left\|G_{e}\left(e^{j \theta}\right)\right\|_{\infty}^{k}\|e(t, 0)\|_{2}
$$

Hence, if:

$$
\left\|G_{e}\left(e^{j \theta}\right)\right\|_{\infty}<1, \forall \theta \in[-\pi, \pi]
$$

is set up, then the tracking error $e(t, k)$ of the system converges monotonically in the sense of $\ell_{2}$ norm.

The controlled dynamics can be written as:

$$
\left\{\begin{array}{l}
\widetilde{\eta}(t+1, k+1)=A_{1} \widetilde{\eta}(t, k+1)+B_{1} e(t, k) \\
e(t, k+1)=C_{1} \widetilde{\eta}(t, k+1)+D_{1} e(t, k)
\end{array}\right.
$$

where:

- $A_{1}=A+B K_{1}$

- $B_{1}=B K_{2}+\left(A+B K_{1}\right) B K_{3}$

- $\quad C_{1}=-C$

- $\quad D_{1}=I-C B K_{3}$

The state-space model (33) is that of a discrete linear repetitive process with pass output and state vector $e(t, k)$ and $\eta(t, k+1)$ respectively, once the initial conditions are specified-that is, the pass state initial vector $\widetilde{\eta}(0, k+1), k \geq 1$, and the initial pass profile $e(t, k), 0 \leq t \leq T-1$.

Stability along the pass of this repetitive process guarantees that the error sequence $\{e(t, k)\}$ converges to zero as $k \rightarrow \infty$ independently of the pass length.

\section{Monotone Convergence Analysis of ILC System in Finite Frequency Domain}

This section discusses the monotone convergence of discrete linear repetition processes in the finite frequency domain. Derive a new stability test formula for linear matrix inequalities.

Routine analysis shows that condition (32) is replaced with:

$$
\left\|G_{e}\left(e^{j \theta}\right)\right\|_{\infty}<\gamma, \forall \theta \in[-\pi, \pi], \gamma \in(0,1]
$$


At this point, Inequality (34) is no longer a standard $H_{\sim}$ control problem. For this reason, according to the method in the literature [20], $\gamma=\varepsilon_{1} \varepsilon_{2}^{-1}$ is defined. For $\varepsilon_{1}>0$ and $\varepsilon_{2}>0$, the conditions are met:

$$
\varepsilon_{1} \leq \varepsilon_{2}
$$

so $\gamma \in(0,1]$ is guaranteed.

At this point, the Inequality (34) can be converted to

$$
\left\|\bar{G}_{e}\left(e^{j \theta}\right)\right\|_{\infty}<\varepsilon_{1}, \forall \omega \in[-\pi, \pi]
$$

where $\bar{G}_{e}\left(e^{j \theta}\right)=\varepsilon_{2} G_{e}\left(e^{j \theta}\right)=C_{1}\left(e^{j \theta} I-A_{1}\right)^{-1} \varepsilon_{2} B_{1}+\varepsilon_{2} D_{1} \forall \theta \in[-\pi, \pi]$.

Therefore, Inequality (36) can be considered as an $H_{\sim}$ control problem constrained by the linear condition (35). The convergence condition (36) can be analysed by the KYP lemma in the finite frequency domain $\theta \in[-\pi, \pi]$, so as to obtain a more practical and relevant method to solve such problems. In Lemma 1, considering the frequency rate response matrix is $\bar{G}_{e}\left(e^{j \theta}\right)$, select $P i$ as:

$$
\Pi=\left[\begin{array}{cc}
\varepsilon_{1}^{-1} I & 0 \\
0 & -\varepsilon_{1} I
\end{array}\right]
$$

The following inequality can be obtained by combining Equation (3):

$$
\bar{G}_{e}\left(e^{j \theta}\right)^{T} * \bar{G}_{e}\left(e^{j \theta}\right)<\varepsilon_{1}^{2} I, \forall \theta \in[-\pi, \pi]
$$

It is obvious that the convergence conditions (36) and (38) are equivalent. The establishment of equation (38) means the tracking error transfer function of the state-space model (33) $G_{e}(z)$ satisfies the third condition of Lemma 1. Then the convergence performance of the PD-type ILC process is analysed in the finite frequency domain.

\subsection{ILC of Nominal Systems}

Theorem 1. Consider the discrete linear repetitive process (1), under the action of PD-type iterative learning control laws (15) $\backsim(17)$, if there exist matrices $Y_{1}, Y_{2}, \hat{S}>0, \hat{Q}>0, \hat{P}=\hat{P}^{T}>0$, an invertible matrix of appropriate dimensions $\hat{W}$, and scalars $\rho_{1}, \rho_{2}, \varepsilon_{2} \geq \varepsilon_{1}>0$, such that the following two LMIs are feasible:

$$
\begin{gathered}
H_{11}=\left[\begin{array}{ccc}
\hat{S}+\rho_{2} \hat{W}+\rho_{2} \hat{W}^{T} & -\rho_{1} \hat{W}-\rho_{2}\left(A \hat{W}+B Y_{1}\right) \\
* & -\hat{S}+\rho_{1} s y m\left(A \hat{W}+B Y_{1}\right)
\end{array}\right]<0 \\
H_{12}=\left[\begin{array}{ccccc}
\hat{\Xi}_{11} & \hat{\Xi}_{12}-\hat{W}^{T} & 0 & 0 & 0 \\
* & \hat{\Xi}_{22}+\operatorname{sym}\left(A \hat{W}+B Y_{1}\right) & B Y_{2} & (-C \hat{W})^{T} & A \hat{W}+B Y_{1} \\
* & * & -\varepsilon_{1} I & \varepsilon_{2} I-\left(C B Y_{3}\right)^{T} & \left(B Y_{3}\right)^{T} \\
* & * & * & -\varepsilon_{1} I & 0 \\
* & * & * & * & -\hat{W}-\hat{W}^{T}
\end{array}\right]<0
\end{gathered}
$$

where $\Xi_{11}, \Xi_{12}$ and $\Xi_{22}$ are the block entries in a matrix Xi with the structure (5), $\rho_{1}$ and $\rho_{2}$ satisfy the following conditions:

$$
\rho_{1}^{2}-\rho_{2}^{2}<0
$$

Hence, within a certain frequency range $\theta \in[-\pi, \pi]$, the systems under ILC are stable along the pass and monotonic pass-to-pass error convergence occur. If these LMIs are feasible, the ILC law matrices are given by:

$$
K_{1}=Y_{1} \hat{W}^{-1}, \quad K_{2}=\varepsilon_{2}^{-1} \Upsilon_{2}, \quad K_{3}=\varepsilon_{2}^{-1} \Upsilon_{3}
$$


Proof of Theorem 1. According to the Lyapunov stability theory of the standard discrete linear repeating process, condition (ii) of Lemma 1 is equivalent to Inequality (43):

$$
\left[\begin{array}{ll}
A_{1}^{T} & I
\end{array}\right]\left[\begin{array}{cc}
S & 0 \\
0 & -S
\end{array}\right]\left[\begin{array}{c}
A_{1} \\
I
\end{array}\right]=A_{1}^{T} S A_{1}-S<0
$$

where $S>0$, set $\Lambda_{1}^{\perp}=\left[\begin{array}{ll}A_{1}^{T} & I\end{array}\right]^{T}, \Lambda_{1}=\left[\begin{array}{ll}-I & A_{1}\end{array}\right]$.

For $\rho_{1}$ and $\rho_{2}$ satisfying Inquation (41), the Inequality (44) is also true:

$$
\left[\begin{array}{ll}
\rho_{1} I & \rho_{2} I
\end{array}\right]\left[\begin{array}{cc}
S & 0 \\
0 & -S
\end{array}\right]\left[\begin{array}{l}
\rho_{1} I \\
\rho_{2} I
\end{array}\right]=\left(\rho_{1}^{2}-\rho_{2}^{2}\right) S<0
$$

set $\Sigma_{1}^{\perp}=\left[\begin{array}{ll}\rho_{1} I & \rho_{2} I\end{array}\right], \Sigma_{1}=\left[\begin{array}{ll}-\rho_{2} I & \rho_{1} I\end{array}\right]$.

According to Lemma 3 , the following equation can be obtained:

$$
\left[\begin{array}{cc}
S & 0 \\
0 & -S
\end{array}\right]+\operatorname{sym}\left\{\left[\begin{array}{c}
-I \\
A_{1}^{T}
\end{array}\right] W\left[\begin{array}{ll}
-\rho_{2} I & \rho_{1} I
\end{array}\right]\right\}<0
$$

where $W$ is solvable, known by the above inequalities $S+\rho_{2} W+\rho_{2} W^{T}<0$ and $W$ is the invertible matrix.

Next post- and pre-multiply (45) by diag $\left\{W^{-1}, W^{-1}\right\}$ and its transpose to obtain (39) on also setting $\hat{W}=W^{-1}$ and $\hat{S}=W \hat{W} T^{S} \hat{W}$.

According to Lemma 2, in the finite frequency domain $\theta \in[-\pi, \pi]$, Inequality (38) is true, which means the existence of the symmetric matrix $P>0$ and the appropriate matrix $Q>0$, which makes the following inequality true:

$$
\left[\begin{array}{ll}
\bar{A}_{1} & \bar{B}_{1} \\
I & 0
\end{array}\right]^{T} \Xi\left[\begin{array}{ll}
\bar{A}_{1} & \bar{B}_{1} \\
I & 0
\end{array}\right]+\left[\begin{array}{cc}
\bar{C}_{1} & \bar{D}_{1}^{T} \\
0 & I
\end{array}\right] \Pi\left[\begin{array}{cc}
\bar{C}_{1} & \bar{D}_{1} \\
0 & I
\end{array}\right]<0
$$

where $\bar{A}_{1}=A_{1}, \bar{B}_{1}=\varepsilon_{2} B_{1}, \bar{C}_{1}=C_{1}, \bar{D}_{1}=\varepsilon_{2} D_{1}, \Xi$ and $\Pi$ are shaped like Equations (5) and (37), respectively.

Further, Equation (45) can be rewritten as:

$$
\left[\begin{array}{ccc}
\bar{A}_{1}^{T} & I & 0 \\
\bar{B}_{1}^{T} & 0 & I
\end{array}\right]\left[\begin{array}{ccc}
\Xi_{11} & \Xi_{12} & 0 \\
* & \Xi_{22}+\varepsilon_{1}^{-1} \bar{C}_{1}^{T} \bar{C}_{1} & \varepsilon_{1}^{-1} \bar{C}_{1}^{T} \bar{D}_{1} \\
* & * & -\varepsilon_{1} I+\varepsilon_{1}^{-1} \bar{D}_{1}^{T} \bar{D}_{1}
\end{array}\right]\left[\begin{array}{cc}
\bar{A}_{1} & \bar{B}_{1} \\
I & 0 \\
0 & I
\end{array}\right]<0
$$

Setting $\left(\Lambda_{2}^{\perp}\right)^{T}=\left[\begin{array}{ccc}\bar{A}_{1}^{T} & I & 0 \\ \bar{B}_{1}^{T} & 0 & I\end{array}\right], \Psi_{2}=\left[\begin{array}{ccc}\Xi_{11} & \Xi_{12} & 0 \\ * & \Xi_{22}+\varepsilon_{1}^{-1} \bar{C}_{1}^{T} \bar{C}_{1} & \varepsilon_{1}^{-1} \bar{C}_{1}^{T} \bar{D}_{1} \\ * & * & -\varepsilon_{1} I+\varepsilon_{1}^{-1} \bar{D}_{1}^{T} \bar{D}_{1}\end{array}\right]$ can be obtained $\Lambda_{2}=\left[\begin{array}{lll}-I & \bar{A}_{1} & \bar{B}_{1}\end{array}\right]$.

In order to apply Lemma 3, select the matrix $\Sigma_{2}=\left[\begin{array}{lll}0 & I & 0\end{array}\right]$. It can be obtained $\left(\Sigma_{2}^{\perp}\right)^{T}=$ $\left[\begin{array}{ccc}I & 0 & 0 \\ 0 & 0 & I\end{array}\right]$, and it satisfies matrix Inequality (48):

$$
\left(\Sigma_{2}^{\perp}\right)^{T} \Psi_{2} \Sigma_{2}^{\perp}=\left[\begin{array}{cc}
\Xi_{11} & 0 \\
* & -\varepsilon_{1} I+\varepsilon_{1}^{-1} \bar{D}_{1}^{T} \bar{D}_{1}
\end{array}\right]<0
$$


Inequality (48) is true if, and only if, its diagonal elements are satisfied as follows:

$$
\left\{\begin{array}{c}
\Xi_{11}<0 \\
-\varepsilon_{1} I+\varepsilon_{1}^{-1} \bar{D}_{1}^{T} \bar{D}_{1}<0
\end{array}\right.
$$

So, $\Xi_{11}=-P<0$ is true, and $-\varepsilon_{1} I+\varepsilon_{1}^{-1} \bar{D}_{1}^{T} \bar{D}_{1}<0$ is equivalent to

$$
D_{1}^{T} D_{1}<\varepsilon_{1} \varepsilon_{2}^{-1}=\gamma
$$

Therefore, $\rho\left(D_{1}\right)<\gamma \leq 1$ is the condition of the progressive convergence of the iterative learning control system, which is naturally valid. Therefore, Equation (48) is obviously valid and satisfies condition (i) of Lemma 1.

When Equation (47) is true, according to Lemma 3, the existence of an invertible matrix $W$ satisfies the following matrix inequality:

$$
\Psi_{2}+\operatorname{sym}\left\{\Lambda_{2}^{T} W \Sigma_{2}\right\}<0
$$

On the basis of the Schur complement lemma, there

$$
\left[\begin{array}{cccc}
\Xi_{11} & \Xi_{12}-W & 0 & 0 \\
* & \Xi_{22}+\operatorname{sym}\left(\bar{A}_{1}^{T} W\right) & W^{T} \bar{B}_{1} & \bar{C}_{1}^{T} \\
* & * & -\varepsilon_{1} I & \bar{D}_{1}^{T} \\
* & * & * & -\varepsilon_{1} I
\end{array}\right]<0
$$

Next, by left and right multiplying (52) by $\operatorname{diag}\left\{\hat{W}^{T}, \hat{W}^{T}, I, I\right\}$ and its transpose, respectively, the following matrix is found to be true:

$$
\left[\begin{array}{cccc}
\hat{\Xi}_{11} & \hat{\Xi}_{12}-\hat{W}^{T} & 0 & 0 \\
* & \hat{\Xi}_{22}+\operatorname{sym}\left(\bar{A}_{1} \hat{W}\right) & \bar{B}_{1} & \left(\bar{C}_{1} \hat{W}\right)^{T} \\
* & * & -\varepsilon_{1} I & \bar{D}_{1}^{T} \\
* & * & * & -\varepsilon_{1} I
\end{array}\right]<0
$$

where $\hat{\Xi}_{11}=\hat{W}^{T} \Xi_{11} \hat{W}, \hat{\Xi}_{12}=\hat{W}^{T} \Xi_{12} \hat{W}, \hat{\Xi}_{22}=\hat{W}^{T} \Xi_{22} \hat{W}, \hat{P}=\hat{W}^{T} P \hat{W}, \hat{Q}=\hat{W}^{T} Q \hat{W}$.

Inequality (53) can be written as

$$
\Omega+J N^{T}+N J^{T}<0
$$

$$
\begin{gathered}
\text { where } \Omega=\left[\begin{array}{cccc}
\hat{\Xi}_{11} & \hat{\Xi}_{12}-\hat{W}^{T} & 0 & 0 \\
* & \hat{\Xi}_{22}+\operatorname{sym}\left(A \hat{W}+B Y_{1}\right) & B Y_{2} & (-C \hat{W})^{T} \\
* & * & -\varepsilon_{1} I & \varepsilon_{2} I-\left(C B Y_{3}\right)^{T} \\
* & * & * & -\varepsilon_{1} I
\end{array}\right] \\
J=\left[\begin{array}{cccc}
0 & \left(A+B K_{1}\right)^{T} & 0 & 0
\end{array}\right]^{T} \\
N^{T}=\left[\begin{array}{llll}
0 & 0 & B Y_{3} & 0
\end{array}\right] \\
Y_{1}=K_{1} \hat{W}
\end{gathered}
$$


Inequality (54) can be written as

$$
\left[\begin{array}{ll}
I & J
\end{array}\right]\left[\begin{array}{ll}
\Omega & N \\
N^{T} & 0
\end{array}\right]\left[\begin{array}{l}
I \\
J^{T}
\end{array}\right]<0
$$

Also, to apply Lemma 3, letting $\Psi_{3}=\left[\begin{array}{ll}\Omega & N \\ N^{T} & 0\end{array}\right],\left(\Sigma_{3}^{\perp}\right)^{T}=\left[\begin{array}{ll}I & J\end{array}\right]$, we have $\Sigma_{3}=$ $\left[\begin{array}{ll}J^{T} & -I\end{array}\right]$.

Select matrix $\Lambda_{3}=\left[\begin{array}{ll}0 & 1\end{array}\right],\left(\Lambda_{3}^{\frac{1}{3}}\right)^{T}=\left[\begin{array}{ll}I & 0\end{array}\right]$. Then there is an invertible matrix $W_{1}=\hat{W}^{T}$, which makes the following inequality true:

$$
\Psi_{3}+\operatorname{sym}\left\{\Lambda_{3}^{T} W_{1} \Sigma_{3}\right\}<0
$$

Inequality (40) can be obtained, hence condition (iii) of Lemma 1 holds and the proof is complete.

\subsection{ILC of Norm-Bounded Uncertainty System}

Furthermore, on the basis of Theorem 1, linear repeated processes (11) are considered to contain norm uncertainty:

$$
\bar{A}=A+\Delta A, \quad \bar{B}=B+\Delta B
$$

Matrices $\Delta A, \Delta B$ denote uncertainties that are assumed to satisfy:

$$
\triangle A=H F E_{1}, \quad \Delta B=H F E_{2}
$$

where $H, E_{1}, E_{2}$ are known real constant matrices of compatible dimensions, and $F$ is an unknow and perturbation satisfying:

$$
F^{T} F \leq I
$$

Similarly, the iterative learning controller (15)-(17) is adopted, and the state-space model (33) becomes:

$$
\left\{\begin{array}{l}
\eta(t+1, k+1)=A_{2} \eta(t, k+1)+B_{2} e(t, k) \\
e(t, k+1)=C_{2} \eta(t, k+1)+D_{2} e(t, k)
\end{array}\right.
$$

where $A_{2}=\bar{A}+\bar{B} K_{1}$

$$
\begin{aligned}
& B_{2}=\bar{B} K_{2}+\left(\bar{A}+\bar{B} K_{1}\right) \bar{B} K_{3} \\
& C_{2}=-C, \quad D_{2}=I-C \bar{B} K_{3} .
\end{aligned}
$$

Theorem 2. Consider the uncertain system under the control of $P D$-type iterative learning (60), if there are matrices $Y_{1}, Y_{2}, \hat{S}>0, \hat{Q}>0, \hat{P}=\hat{P}^{T}>0$, an invertible matrix of appropriate dimensions $\hat{W}$ and scalars $\rho_{1}$, $\rho_{2}, \varepsilon_{2} \geq \varepsilon_{1}>0, \lambda_{1}>0, \lambda_{2}>0$, such that the following two LMIs are feasible:

$$
\begin{aligned}
H_{21} & =\left[\begin{array}{ccc}
H_{11} & \lambda_{1} X_{1} & N_{1}^{T} \\
* & -\lambda_{1} I & 0 \\
* & * & -\lambda_{1} I
\end{array}\right]<0 \\
\mathrm{H}_{22} & =\left[\begin{array}{ccc}
\mathrm{H}_{12} & \lambda_{2} X_{2} & N_{2}^{T} \\
* & -\lambda_{2} I & 0 \\
* & * & -\lambda_{2} I
\end{array}\right]<0
\end{aligned}
$$


where $H_{11}$ and $H_{12}$ are shown in Theorem 1:

$$
\begin{gathered}
X_{1}=\left[\begin{array}{lll}
-\left(\rho_{2} H\right)^{T} & \left(\rho_{1} H\right)^{T}
\end{array}\right]^{T} \\
X_{2}=\left[\begin{array}{ccccc}
0 & H^{T} & 0 & 0 & 0 \\
0 & 0 & 0 & -(C H)^{T} & H^{T}
\end{array}\right]^{T} \\
N_{1}=\left[\begin{array}{lll}
0 & E_{1} \hat{W}+E_{2} Y_{1}
\end{array}\right] \\
N_{2}=\left[\begin{array}{ccccc}
0 & E_{1} \hat{W}+E_{2} Y_{1} & E_{2} Y_{2} & 0 & E_{1} \hat{W}+E_{2} Y_{1} \\
0 & 0 & E_{2} Y_{3} & 0 & 0
\end{array}\right]
\end{gathered}
$$

Therefore, within a finite certain frequency range $\theta \in[-\pi, \pi]$, the system under iterative learning control is robustly stable along the pass and monotonic pass-to-pass error convergence occurs. Then the gain matrix of the iterative learning control law can be obtained, as shown in Equation (42).

Proof of Theorem 2. Let's prove that the inequality $H_{21}<0$ is true, and $H_{22}<0$ can be proved by the same method. By adding uncertainty on the basis of Theorem $1, H_{21}<0$ can be written as:

$$
H_{21}=H_{11}+\operatorname{sym}\left\{X_{1} F N_{1}\right\}<0
$$

by Lemma 4 . The following inequality is feasible if, and only if, scalar $\lambda_{1}>0$ exists:

$$
H_{11}+\lambda_{1} X_{1} X_{1}^{T}+\lambda_{1}^{-1} N_{1}^{T} N_{1}<0
$$

The application of Schur's complement formula to (64) gives:

$$
\left[\begin{array}{cc}
\mathrm{H}_{11} & {\left[\begin{array}{cc}
\lambda_{1}^{1 / 2} X_{1} & \lambda_{1}^{-1 / 2} N_{1}^{T}
\end{array}\right]} \\
* & -I
\end{array}\right]<0
$$

Finally, $H_{21}<0$ in Inequality (61) can be proved by multiplying left and right by diagonal matrix $\operatorname{diag}\left\{I, I, \lambda_{1}^{1 / 2} I, \lambda_{1}^{1 / 2} I\right\}$ respectively. Similarly, $H_{22}<0$ in Equation (62) can be obtained. Theorem 2 is proved.

\subsection{ILC of Polyhedron Uncertainty System}

The model matrices A and B considering (11) are not clear, but belong to the polytope type uncertainty domain denoted as $D_{m}$. So if think backwards, it means that any uncertain matrix can be written as a convex combination of the vertices:

$$
D_{m}=\left\{(A(\xi), B(\xi)) \mid(A(\xi), B(\xi))=\sum_{i=1}^{N} \xi_{i}\left(A_{i}, B_{i}\right), \xi_{i} \geq 0, \sum_{i=1}^{N} \xi_{i}=1\right\}
$$

In this representation, $N$ denotes the corresponding number of vertices, the matrices $A_{i}$ and $B_{i}$ define the vertices of a polytope in which the actual matrices $A$ and $B$ are assumed to lie.

With uncertain parameters, the linear repeating process (11) described in Equation (66) can be obtained. Under the action of PD-type iterative learning control, the state-space model obtained can be:

$$
\left\{\begin{array}{l}
\widetilde{\eta}(t+1, k+1)=A_{3}(\widetilde{\xi}) \widetilde{\eta}(t, k+1)+B_{3}(\xi) e(t, k) \\
e(t, k+1)=C_{3}(\xi) \widetilde{\eta}(t, k+1)+D_{3}(\xi) e(t, k)
\end{array}\right.
$$


where

$$
\begin{aligned}
A_{3}(\xi) & =A(\xi)+B(\xi) K_{1} \\
B_{3}(\xi) & =B(\xi) K_{2}+\left(A(\xi)+B(\xi) K_{1}\right) B(\xi) K_{3} \\
C_{3}(\xi) & =-C, \quad D_{3}(\xi)=I-C B(\xi) K_{3}
\end{aligned}
$$

The model described by (67), where the process matrix is affected by the uncertainty in (66). The model is require to be stable in the channel process if and only if, for any value of $\xi$ in $D_{m}$.

Suppose that the required matrix functions $\hat{P}(\xi), \hat{Q}(\xi)$ and $\hat{S}(\xi)$ are explicit and have the following form:

$$
\hat{P}(\xi)=\sum_{i=1}^{N} \xi_{i} \hat{P}_{i}, \hat{Q}(\xi)=\sum_{i=1}^{N} \xi_{i} \hat{Q}_{i}, \hat{S}(\xi)=\sum_{i=1}^{N} \xi_{i} \hat{S}_{i}
$$

The matrices defined in (68) are parameter-dependent Lyapunov functions and can take $N$ different values corresponding to the vertices of the polytope $D_{m}$.

According to parameter-dependent Lyapunov functions $\hat{P}(\xi)$ and $\hat{Q}(\xi)$ of the form (68), matrices $\hat{\Xi}_{11 i}, \hat{\Xi}_{12 i}$ and $\hat{\Xi}_{22 i}$ have affine form:

$$
\hat{\Xi}_{11}(\xi)=\sum_{i=1}^{N} \xi_{i} \hat{\Xi}_{11}, \hat{\Theta}_{12}(\xi)=\sum_{i=1}^{N} \xi_{i} \hat{\Theta}_{12 i}, \hat{\Xi}_{22}(\xi)=\sum_{i=1}^{N} \xi_{i} \hat{\Theta}_{22 i}
$$

Theorem 3 (learning (67)). If there are matrices $Y_{1}, Y_{2}, \hat{S}_{t}>0, \hat{Q}_{i}>0, \hat{P}_{i}=\hat{P}_{i}^{T}>0$, the invertible matrix $\hat{W}$ with the appropriate dimensions, scalars $\rho_{1}, \rho_{2}, \varepsilon_{2} \geq \varepsilon_{1}>0$, the following linear matrix inequalities are feasible for all $i=1,2, \cdots, N$

$$
\begin{gathered}
H_{31 i}=\left[\begin{array}{ccc}
\hat{S}_{i}+\rho_{2} \hat{W}+\rho_{2} \hat{W}^{T} & -\rho_{1} \hat{W}-\rho_{2}\left(A_{i} \hat{W}+B_{i} Y_{1}\right) \\
* & -\hat{S}_{i}+\rho_{1} \operatorname{sym}\left(A_{i} \hat{W}+B_{i} Y\right)
\end{array}\right]<0 \\
H_{32 i}=\left[\begin{array}{ccccc}
\hat{\Xi}_{11 i} & \hat{\Xi}_{12 i}-\hat{W}^{T} & 0 & 0 & 0 \\
* & \hat{\Xi}_{22 i}+\operatorname{sym}\left(A_{i} \hat{W}+B_{i} Y_{1}\right) & B_{i} Y_{2} & (-C \hat{W})^{T} & A_{i} \hat{W}+B_{i} Y_{1} \\
* & * & -\varepsilon_{1} I & \varepsilon_{2} I-\left(C B_{i} Y_{3}\right)^{T} & \left(B_{i} Y_{3}\right)^{T} \\
* & * & * & -\varepsilon_{1} I & 0 \\
* & * & * & * & -\hat{W}-\hat{W}^{T}
\end{array}\right] \\
<0
\end{gathered}
$$

where $\hat{\Xi}_{11 i}, \hat{\Xi}_{12 i}, \hat{\Xi}_{22 i}$ are shaped as Equation (69), $\rho_{1}$ and $\rho_{2}$ satisfy condition (41). Then, in a certain finite frequency range $\theta \in[-\pi, \pi]$ the system under the control of iterative learning is robust and stable along the pass-to-pass direction, and the tracking error can be monotonically converged. Hence, the gain matrix of the ILC law can be obtained, as shown in Equation (42).

Proof of Theorem 3. For each vertex of polyhedron matrix, the proof process is the same as Theorem 1.

\section{Simulation}

In this section, an example is given to verify the proposed ILC scheme in a finite frequency domain. Considering the injection velocity in injection molding [21], the injection speed was considered as the research object. This injection molding process [21] is a typical iterative system, because the controlled variables should be followed certain profiles to make sure the process quality. The dynamic equation is described as follows:

$$
\left\{\begin{array}{l}
x(t+1, k)=\left[\begin{array}{cc}
1.582 & -0.5916 \\
1 & 0
\end{array}\right] x(t, k)+\left[\begin{array}{l}
1 \\
0
\end{array}\right] u(t, k) \\
y(t, k)=\left[\begin{array}{ll}
1.69 & 1.419
\end{array}\right] x(t, k)
\end{array}\right.
$$


The expected trajectory of the system is defined as:

$$
y_{d}(t)=\left\{\begin{array}{cc}
\sin (0.01 \pi t) & 0 \leq t<200 \\
1 & 200 \leq t<300 \\
4-0.01 t & 300 \leq t \leq 400
\end{array}\right.
$$

The sampling time is set as $T_{s}=0.1 \mathrm{~s}$. Its reference trajectory is shown in Figure $1 \mathrm{a}$, and the spectrum obtained by FFT is shown in Figure 1b. It can be seen from the figure that the effective harmonic of the reference track varies from 0 to $5 \mathrm{~Hz}$, which is in the low-frequency range. Therefore, $\theta=0.3142$.

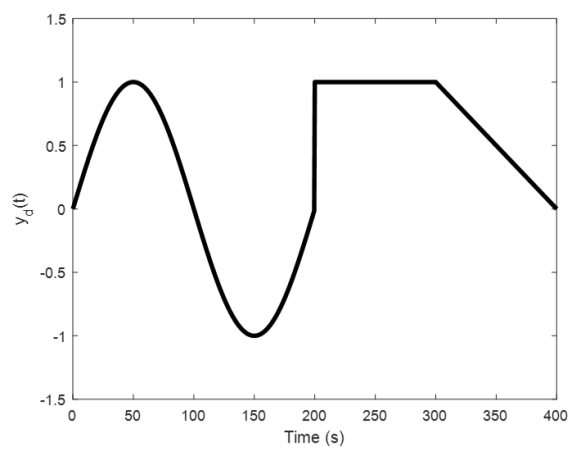

(a) Output reference track

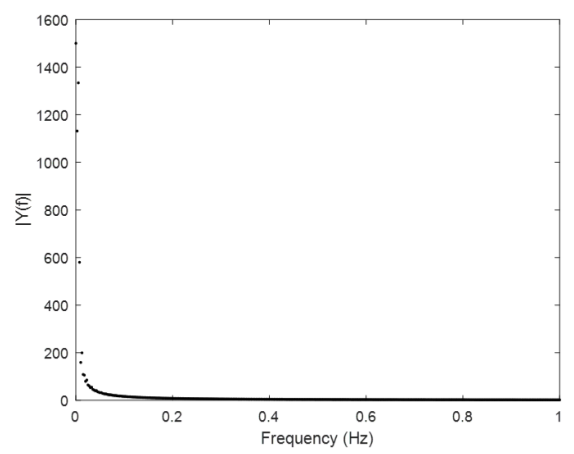

(b) The spectrum of the output reference trajectory

Figure 1. Output reference track and the spectrum of the output reference trajectory.

And it is assumed that the initial state for each trial is 0 . Also, pass-to-pass error convergence is measured by the root mean square formula:

$$
R M S=\sqrt{\frac{1}{T} \sum_{t=1}^{T} e^{2}(t, k)}
$$

In order to compare the effect of the state feedback PD-type ILC method and the state feedback P-type iterative learning control scheme, the corresponding state feedback P-type iterative learning control law is designed under the same condition as shown below:

$$
u(t, k+1)=u(t, k)+L_{1} \eta(t+1, k+1)+L_{2} e(t+1, k)
$$

\subsection{Case 1 Norm-Bounded Uncertainty}

In this case, considering that the linear repeated process (11) has an uncertainty of form (57), the parameters are shown as follows: $H=\left[\begin{array}{ll}0.1 & 0 \\ 0 & 0.1\end{array}\right], E_{1}=\left[\begin{array}{ll}0.1 & 0 \\ -0.1 & 0\end{array}\right], E_{2}=\left[\begin{array}{l}0.02 \\ 0.02\end{array}\right]$ with uncertain $F$ taking its varies between $-1 \sim 1$. Selecting $\rho_{1}=2$ and $\rho_{2}=-5$, the following parameters can be obtained by using Theorem 2: $K_{1}=\left[\begin{array}{ll}-1.9061 & 0.4426\end{array}\right], K_{2}=0.1520, K_{3}=0.3119$.

The parameters of the state feedback P-type iterative learning controller are obtained by the same method: $L_{1}=[-1.8410-0.4643], L_{2}=0.3197$.

Figure 2 shows the changed surface of tracking error with iteration time and batch. It can be seen that the state feedback PD-type iterative learning control system can achieve good control performance in time and iterative batch direction when there is norm-bounded uncertainty interference. 


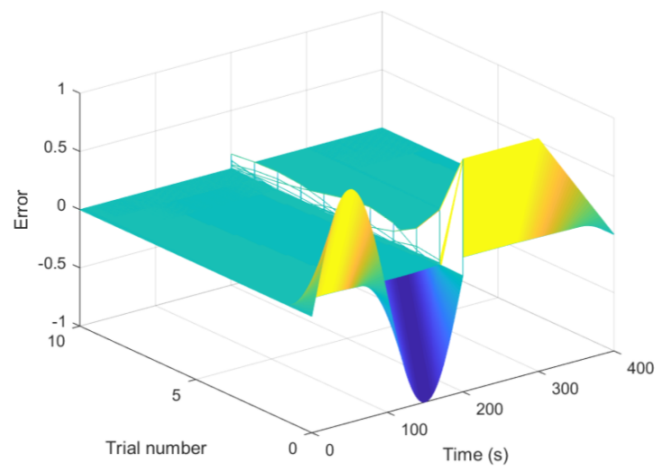

Figure 2. Surface variation of tracking error of norm-bounded uncertainty system with time and iteration times.

In Figure 3, the curve of the RMS error of the system with the number of iterations under the action of the two control laws is given. All of these show that the tracking errors of norm-bounded uncertainty systems converge monotonically under the action of two control laws, and the system has better convergence performance under the action of state feedback PD type iterative learning control.

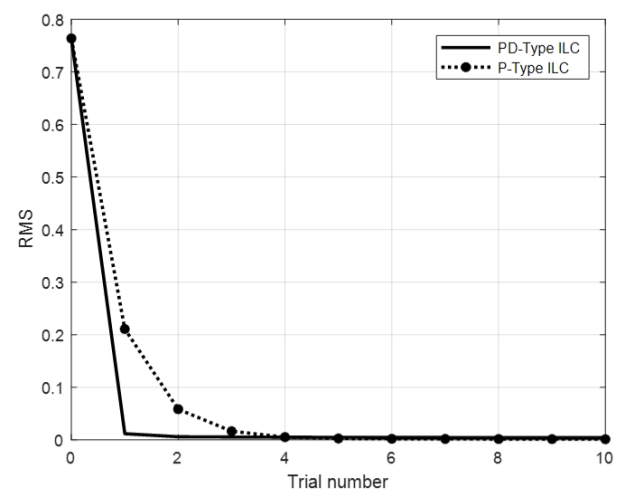

Figure 3. Curve of RMS of norm-bounded uncertain system with the number of iterations.

\subsection{Case 2 Polyhedron Uncertainty}

In this case, the polyhedral uncertainty of the system parameter matrix is considered as:

$$
\left\{\begin{array}{l}
A(\xi)=\xi_{1} A^{1}+\xi_{2} A^{2} \\
B(\xi)=\xi_{1} B^{1}+\xi_{2} B^{2}
\end{array}\right.
$$

where, $\xi_{1}$ and $\xi_{2}$ are between [0,1], which meet the requirement of $\xi_{1}+\xi_{2}=1$. Moreover, the convex optimization method is used to avoid solving the innumerable points, and the infinite dimensional solution problem is transformed into the convex optimization problem of the vertices.

The vertex matrix of a convex polyhedral uncertain system is:

$$
\begin{aligned}
& \text { Vertex } 1: A^{1}=\left[\begin{array}{cc}
1.584 & -0.5916 \\
1.01 & 0
\end{array}\right], B^{1}=\left[\begin{array}{l}
1.02 \\
0.01
\end{array}\right] \\
& \text { Verter } 2: A^{2}=\left[\begin{array}{cc}
1.580 & -0.5916 \\
0.99 & 0
\end{array}\right], B^{2}=\left[\begin{array}{l}
0.98 \\
-0.01
\end{array}\right]
\end{aligned}
$$


Setting $\rho_{1}=1, \rho_{2}=10$, the following parameter values can be obtained by using Theorem 2 :

$$
K_{1}=\left[\begin{array}{ll}
-2.5805 & 0.2702
\end{array}\right], \quad K_{2}=0.0654, \quad K_{3}=0.3205
$$

Similarly, the state feedback P-type iterative learning controller parameters can be obtained as:

$$
L_{1}=\left[\begin{array}{ll}
-2.3683 & 0.4076
\end{array}\right], \quad L_{2}=0.2036
$$

Figure 4 shows the changed surface of tracking error with iteration time and batch, indicating the control performance of polyhedral uncertainty system in time and iterative batch direction under the state feedback PD-type iterative learning control.

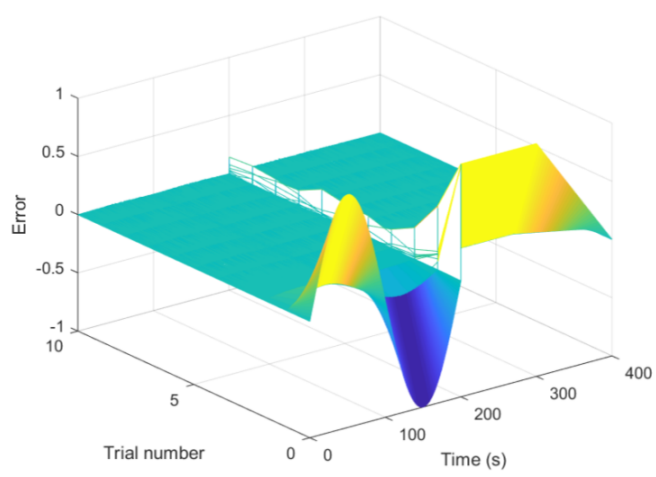

Figure 4. Surface of tracking error of polyhedron uncertainty system varying with time and iteration times.

In Figure 5 shows the two kinds of control law system under the action of the root mean square of the tracking error curve, along with the change of the number of iterations can be seen from the diagram, polyhedron uncertainty system under the action of two kinds of control law has better convergence performance, and the state feedback PD type iterative learning control system under the action of convergence and robustness is superior.

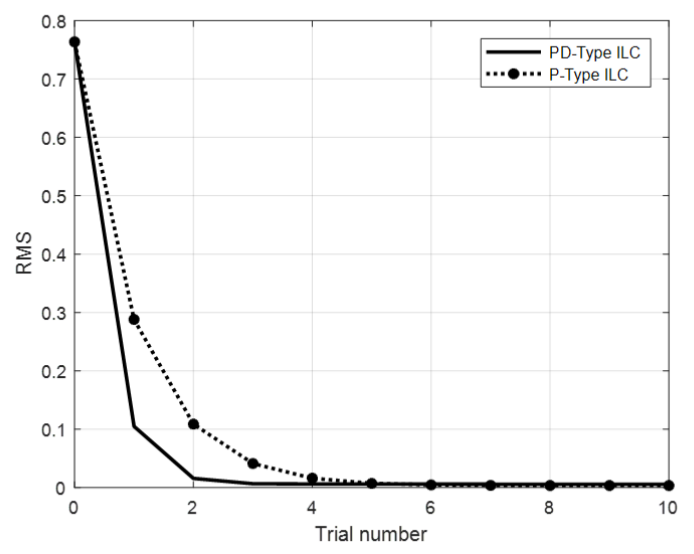

Figure 5. Curve of RMS of polyhedron uncertain system with the number of iterations.

\section{Conclusions}

Based on the stability theory of discrete linear repetitive processes, this paper studied the PD-type iterative learning control problem of state feedback for discrete linear repetitive processes in the limited frequency domain. Firstly, an integrated state feedback PD-type iterative learning controller in finite frequency domain was designed. By using the generalised KYP lemma, sufficient conditions for the existence of the controller and the gain matrix of the controller were given in the form of LMI. At 
the same time, the condition guarantees the dynamic performance of the system. Furthermore, the norm-bounded and convex polyhedral uncertainties of the system model matrix were considered. In the simulation of the injection velocity in injection molding, the state feedback P-type iterative learning control algorithm was compared, which verifies the effectiveness and superiority of the proposed design method.

Author Contributions: Conceptualization, L.W. and H.Y.; methodology, L.W.; validation, L.W.; formal analysis, L.W. and M.L.; data curation, L.W.; writing-original draft preparation, L.W. and H.Y.; writing-review and editing, M.L.; supervision, H.Y.; project administration, H.Y.; funding acquisition, H.Y. All authors have read and agreed to the published version of the manuscript.

Funding: This research was funded by National Natural Science Foundation of China grant number 61773181, 111 Project in China grant number B12018 and Fundamental Research Funds for the Central Universities in China grant number JUSRP51733B.

Conflicts of Interest: The authors declare no conflict of interest.

\section{References}

1. Arimoto, S.; Kawamura, S.; Miyazaki, F. Bettering operation of robots by learning. J. Robot. Syst. 1984, 1, 123-140. [CrossRef]

2. Bristow, D.; Tharayil, M.; Alleyne, A. Survey of iterative learning control: A learning-based method for high-performance tracking control. IEEE Control Syst. 2006, 26, 96-114.

3. Chen, W.; Tomizuka, M. Dual-stage iterative learning control for mimo mismatched system with application to robots with joint elasticity. IEEE Trans. Control Syst. Technol. 2014, 22, 1350-1361.

4. Mandra, S.; Galkowski, K.; Aschemann, H. Robust guaranteed cost ilc with dynamic feedforward and disturbance compensation for accurate pmsm position control. Control Eng. Pract. 2017, 65, 36-47. [CrossRef]

5. Hao, S. Two-dimensional delay compensation based iterative learning control scheme for batch processes with both input and state delays. J. Frankl. Inst. 2019, 356, 8118-8137. [CrossRef]

6. Chen, Y.; Chu, B.; Freeman, C.T.; Liu, Y. Generalized iterative learning control with mixed system constraints: A gantry robot based verification. Control Eng. Pract. 2020, 95, 104260. [CrossRef]

7. Galkowski, K.; Rogers, E.; Xu, S.; Lam, J.; Owens, D.H. Lmis-A fundamental tool in analysis and controller design for discrete linear repetitive processes. IEEE Trans. Circuits Syst. I: Fundam. Theory Appl. 2002, 49, 768-778. [CrossRef]

8. Maleki, S.; Rapisarda, P.; Rogers, E. Failure identification for linear repetitive processes. multidim syst sign process. Multidimens. Syst. Signal Process. 2015, 26, 1037-1059. [CrossRef]

9. Shi, J.; Zhou, H.; Cao, Z.; Jiang, Q. A design method for indirect iterative learning control based on two-dimensional generalized predictive control algorithm. J. Process Control 2014, 24, 1527-1537. [CrossRef]

10. Hladowski, L.; Galkowski, K.; Cai, Z.; Rogers, E.; Freeman, C.T.; Lewin, P.L. A 2d systems approach to iterative learning control for discrete linear pro-cesses with zero markov parameters. Int. J. Control 2011, 84, 1246-1262. [CrossRef]

11. Ding, J.; Cichy, B.; Galkowski, K.; Rogers, E.; Yang, H. Parameter-dependent lyapunov function-based robust iterative learning control for discrete systems with actuator faults. Int. J. Adapt. Control Signal Process. 2016, 30, 1714-1732. [CrossRef]

12. Hongfeng, T.; Yan, L.; Huizhong, Y. Output information based fault-tolerant iterative learning control for dual-rate sampling process with disturbances and output delay. Math. Probl. Eng. 2018. [CrossRef]

13. Paszke, W. Finite frequency control of discrete linear repetitive processes with application in iterative learning control. In Proceedings of the 2010 15th International Conference on Methods and Models in Automation and Robotics, Miedzyzdroje, Poland, 23-26 August 2010; pp. 132-137.

14. Paszke, W.; Rogers, E.; Galkowski, K. Robust finite frequency design of itera-tive learning control schemes. IFAC-PapersOnLine 2016, 49, 169-174. [CrossRef]

15. Iwasaki, T.; Hara, S. Feedback control synthesis of multiple frequency do-520 main specifications via generalized kyp lemma. Int. J. Robust Nonlinear Control 2007, 17, 415-434. [CrossRef]

16. Rogers, E.; Galkowski, K.; Owens, D.H. Control Systems Theory and Applications for Linear Repetitive Processes; Springer Science \& Business Media: Berlin/Heidelberg, Germany, 2007; Volume 349. 
17. Boyd, S.; El Ghaoui, L.; Feron, E.; Balakrishnan, V. Linear Matrix Inequalities in System and Control Theory; Siam: Philadelphia, PA, USA, 1994; Volume 15.

18. Xie, L. Output feedback h control of systems with parameter uncertainty. Int. J. Control 1996, 63, 741-750. [CrossRef]

19. Bristow, D.A.; Tharayil, M.; Alleyne, A.G. A survey of iterative learning control. IEEE Control Syst. Mag. 2006, 26, 96-114.

20. Meng, D.; Jia, Y.; Du, J.; Yu, F. Robust learning controller design for mimo stochastic discrete-time systems: An h-based approach. Int. J. Adapt. Control Signal Process. 2011, 25, 653-670. [CrossRef]

21. Shi, J.; Gao, F.R.; Wu, T.J. Robust design of integrated feedback and iterative learning control of a batch process based on a 2D Roesser system. J. Process Control 2005, 15, 907-924. [CrossRef]

(C) 2020 by the authors. Licensee MDPI, Basel, Switzerland. This article is an open access article distributed under the terms and conditions of the Creative Commons Attribution (CC BY) license (http:/ / creativecommons.org/licenses/by/4.0/). 